\section{Pierre Lepage Philippe Gros}

Remerciements

Les auteurs tiennent à remercier Lucille Beaudet et Martine Brault pour leurs critiques pendant la rédaction du manuscrit, de même que Silvia Vidal pour le dessin de la figure 1 et Stephan Ruetz pour la figure 3.

\section{ADRESSE}

P. Lepage: docteur ès sciences. P. Gros : professeur. Département de biochimie, Université McGill, 3655 Drummond, room 907, Montréal, Québec, H3G lY6, Canada.

$\mathrm{m} / \mathrm{s} n^{\circ} 3$, vol. 11, mars 95

\title{
La glycoprotéine $\boldsymbol{P}$ : de la résistance croisée aux médicaments au transport des lipides biliaires
}

La résistance multiple des cellules tumorales aux agents antinéoplasiques est associée à la surexpression d'une protéine membranaire, la glycoprotéine $P$ (P-gp). L'implication de la P-gp dans la résistance pléiotropique aux agents anticancéreux a été clairement démontrée in vitro, quoique son mécanisme d'action reste encore mal compris. Sa structure moléculaire, modélisée à partir de sa séquence primaire, suggère qu'elle agit comme une pompe membranaire dépendante de l'ATP. Localisée à la face luminale des organes émonctoires et à la barrière hémato-encéphalique, sa fonction normale semble reliée à la détoxication. Son isoforme Mdr2, localisée à la membrane des canalicules biliaires, est impliquée dans la translocation de la phosphatidylcholine entre les deux feuillets de la membrane plasmique. La P-gp appartient à la famille des transporteurs membranaires $A B C$ qui inclut STE6, le transporteur du facteur a, impliqué dans la conjugaison de la levure, le canal chlorure CFTR anormal dans la mucoviscidose et les transporteurs de peptides antigéniques TAP1 et TAP2. L'étude combinée de cette famille peut contribuer à mieux comprendre le mécanisme d'action de la P-gp lors du flux sortant des médicaments antitumoraux.

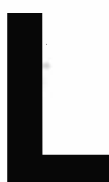

'apparition de cellules résistantes aux agents anticancéreux demeure l'un des obstacles majeurs au traitement de plusieurs types de cancers par la chimiothérapie. Ce phénomène de résistance croisée aux médicaments est beaucoup mieux connu in vitro, où il a été caractérisé à partir de lignées cellulaires résistantes sélectionnées par une exposition continue à un agent antinéoplasique.

\section{Le phénotype de la résistance croisée aux médicaments}

Ces lignées résistantes partagent plusieurs des caractéristiques des cellules tumorales résistantes: elles acquièrent, en plus d'une résistance accrue à l'agent sélectif, une résistance croisée à un certain nombre d'agents cytotoxiques utilisés en chimiothérapie. L'ensemble de ces sub- 


\section{RÉFÉRENCES}

1. Gottesman MM, Pastan I. Biochemistry of multidrug resistance mediated by the multidrug transporter. Annu Rev Biochem 1993; 62: $385-427$

2. Chan HSL, Deboer G, Thorner PS, Haddad G, Gallie BL, Ling Y. Multidrug resistance: clinical opportunities in diagnosis and circumvention. Hematol Oncol Clin North Am $1994 ; 8: 383410$.

3. Marie J. Le phénomène de résistance multiple aux anticancéreux: les gènes $M D R$ et la P-gp. médecine/sciences 1990;6: 443-8.

4. Gros P, Buschman E. The mouse multidrug resistance gene family: structural and functional analysis. International review of cytology. A survey of cell biology. In : Friedlander M, Mueckler M, eds. Molecular biology of receptors and transporters. Pumps, transporters and channels, vol. 137 C. San Diego: Academic Press Inc, 1993: 169-97.

5. Zhang JT, Duthie M, Ling V. Membrane topology of the N-terminal half of the hamster P-glycoprotein molecule. I Biol Chem ster P-glycoprotein

6. Skach WR, Calayag MC, Lingappa VR. Evidence for an alternate model of human P-glycoprotein structure and biogenesis. $J$ Biol Chem 1993; 268 : 6903-8.

7. Shapiro AB, Ling V. ATPase activity of purified and reconstituted P-glycoprotein from Chinese hamster ovary cells. I Biol Chem 1994; 269 : 3745-54.

8. Azzaria M, Schurr E, Gros P. Discrete mutations introduced in the predicted nucleotide-binding sites of the $m d r l$ gene abolish its ability to confer multidrug resistance. Mol Cell Biol 1989; 9 : 5289-97.

9. Bruggemann EP, Currier SJ, Gottesman MM, Pastan I. Characterization of the azidopine and vinblastine binding site of $P$ glycoprotein. J Biol Chem 1992; 267: 210206.

10. Greenberger LM. Major photoaffinity drug labelling sites for iodoaryl azidoprazosin in P-glycoprotein are within, or immediately C-terminal to, transmembrane domains 6 and 12 . J Biol Chem 1993; 268: mains 6

11. Currier SI, Kane SE, Willingham MC, Cardarelli CO, Pastan I, Gottesman MM. Identification of residues in the first cytoplasmic loop of P-glycoprotein involved in the function of chimeric human MDR1MDR2 transporters. I Biol Chem 1992; 667 : 25153-9.

12. Choi K, Chen CJ, Kriegler M, Roninson IB. An altered pattern of cross-resistance in multidrug-resistant human cells results

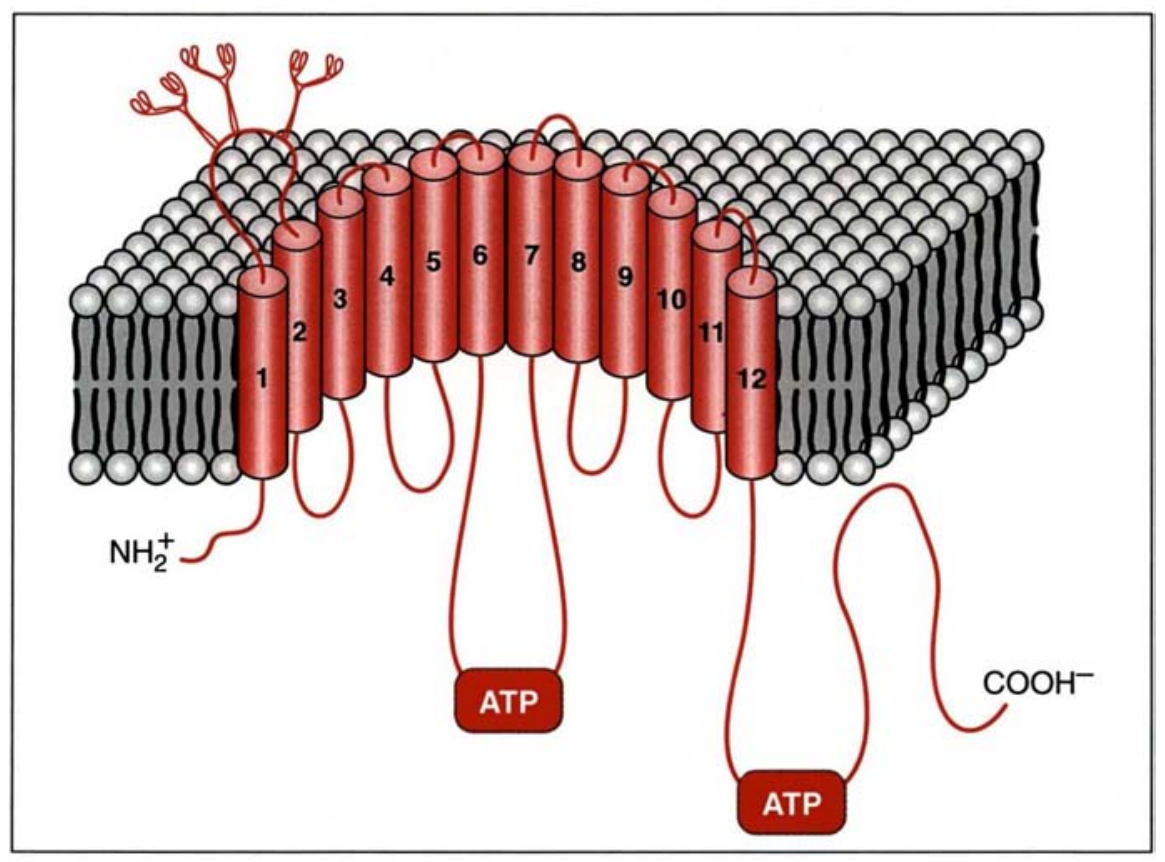

Figure 1. Représentation tridimensionnelle de la glycoprotéine $\boldsymbol{P}$ dans la membrane plasmique. La protéine est formée de deux portions symétriques, chacune incluant six segments transmembranaires (TM) (cylindres numérotés de 1 à 12) et deux sites de fixation de l'ATP (ATP). La première boucle extracellulaire contient trois sites de glycosylation. Les extrémités $\mathrm{NH} 2$ et $\mathrm{COOH}$ terminales sont localisées dans le cytoplasme.

strats de la P-gp (médicaments MDR pour multidrug resistance) inclut des composés lipophiles de type anthracycline (daunomycine et adriamycine), des étoposides et des alcaloïdes de pervenche (vincristine et vinblastine) qui ne présentent entre eux que peu ou pas de similarités de structure et d'activité. La résistance croisée aux médicaments MDR est due à la diminution de l'accumulation intracellulaire de ces médicaments de même qu'à un accroissement de leur expulsion hors de la cellule. Ces deux activités sont associées à une hydrolyse de l'ATP. Enfin, la résistance des cellules tumorales, ainsi que celle des lignées résistantes sélectionnées in vitro, est associée à l'expression d'une phosphoglycoprotéine membranaire de $170 \mathrm{kDa}$, appelée la glycoprotéine P (ou P-gp pour P-glycoprotein) [1-3]. Celle-ci est codée par une famille de gènes très homologues comprenant deux gènes chez l'homme, $M D R 1$ et $M D R 2$ (aussi appelé $M D R 3$ ) [1], et trois chez les rongeurs, dont les gènes murins $m d r l$ (ou $m d r l b$ ), $m d r 2$ et $m d r 3$ (ou $m d r l a$ ) [4].

\section{Structure et fonction de la glycoprotéine $P$}

La séquence d'acides aminés de la protéine P-gp, déduite de la séquence nucléotidique des différents gènes $m d r$, a permis d'établir un modèle structural de la protéine. Celle-ci est formée de deux parties symétriques provenant vraisemblablement de la duplication d'un gène ancestral. Le profil d'hydropathie montre que chacune de ces deux portions contient un domaine hydrophobe composé de six segments transmembranaires (TM) et un domaine hydrophile incluant des motifs Walker* A et B qui révèlent la présence d'un site de fixation de l'ATP (figure 1). La topologie de la région hydrophobe consistant en six segments TM reste cependant controversée et quelques modèles alternatifs suggérant la présence de seulement quatre segments TM ont été proposés (figure 2) [5, 6]. * Courtes séquences en acides aminés, conservées nucléotides. 


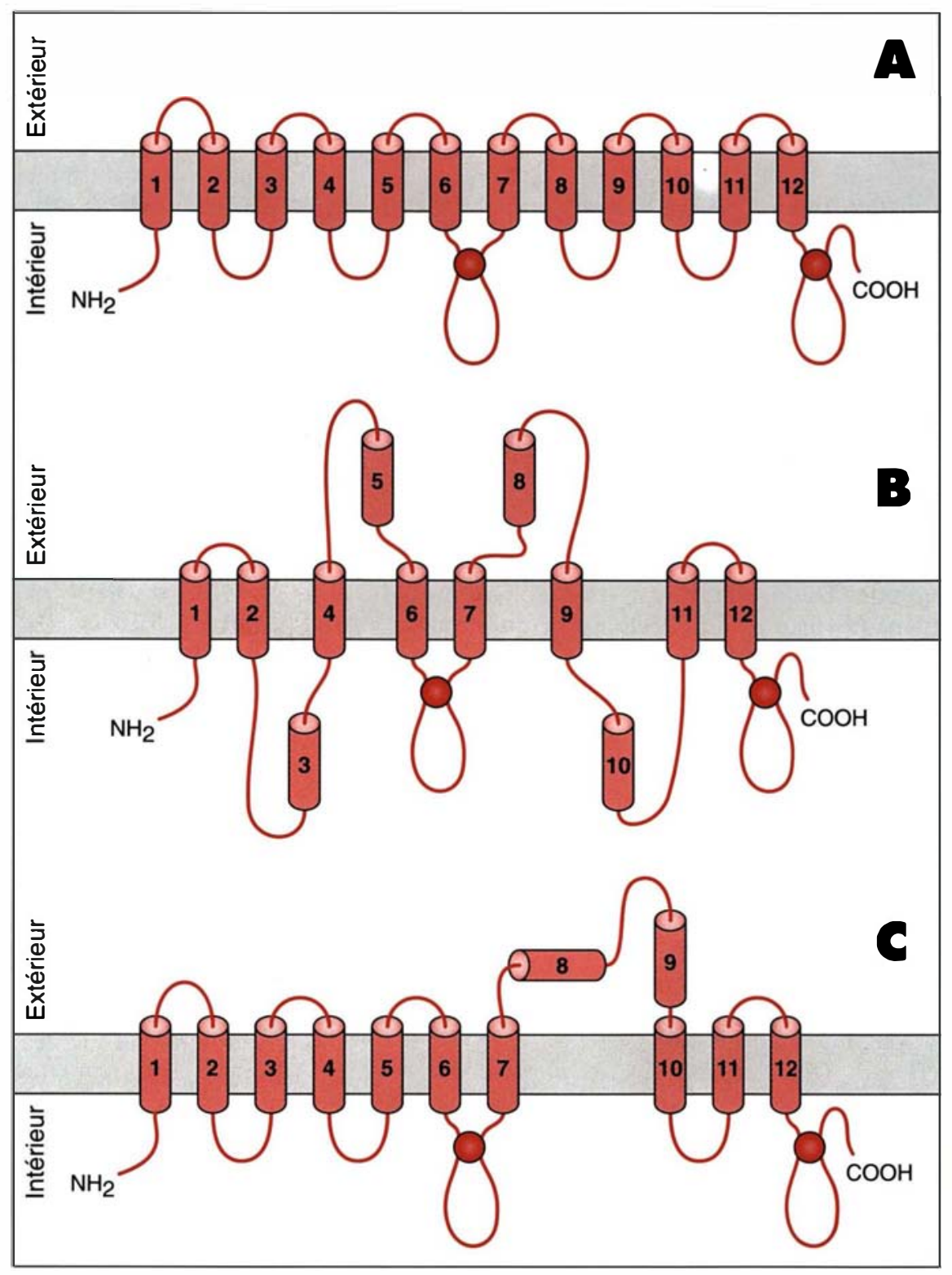

L'implication des gènes $m d r$ dans la résistance cellulaire aux médicaments a été formellement démontrée par transfection $\left(\mathrm{m} / \mathrm{s} \mathrm{n}^{\circ} 10\right.$, vol. 5, p. 790). Ainsi, l'expression des gènes murins $m d r l$ et $m d r 3$ [4], de même que celle du gène humain $M D R 1$ [1], dans des cellules sensibles aux médicaments permet l'acquisition d'une résistance multiple. Des expériences similaires ont toutefois démontré que les gènes murin $m d r 2$ et humain MDR2 ne sont pas impliqués dans le phénotype de résistance croisée aux médicaments. Même si le mécanisme exact d'excrétion des agents antitu$\mathrm{m} / \mathrm{s} n^{\circ} 3$, vol. 11, mars 95 moraux n'est pas encore élucidé, plusieurs observations expérimentales indiquent que la P-gp agirait comme une pompe membranaire dépendante de l'ATP. Premièrement, la surexpression de la P-gp cause une réduction de l'accumulation intracellulaire des médicaments et en augmente l'excrétion. Ces activités peuvent être bloquées par la déplétion de l'ATP intracellulaire ou par des mutations ponctuelles des séquences conservées dans chacun des deux sites de fixation de l'ATP. Deuxièmement, diverses expériences de photomarquage, utilisant des analogues photo-
Figure 2. Modèles de la structure de la glycoprotéine $\boldsymbol{P}$. Représentation schématique de la $P$-gp dans la membrane plasmique. Diverses expériences in vitro ont permis d'élaborer des modèles de la structure de la P-gp distincts du modèle initial (A). B: Modèle proposé par le groupe de Ling [5]. C: Modèle proposé par le groupe de Lingappa [6]. activables des agents anticancéreux, démontrent que ceux-ci se lient directement à la P-gp. Troisièmement, des mutations ponctuelles dans divers segments transmembranaires changent la spécificité de la P-gp pour différents médicaments MDR, en modifiant l'affinité de ces médicaments pour le transporteur.

La reconnaissance et l'excrétion par la P-gp d'une grande variété de composés chimiques de structure et de fonction diverses demeure l'un des aspects les plus énigmatiques de la fonction de cette protéine. L'ensemble des connaissances biochi- 


\section{RÉFÉRENCES}

13. Safa AR, Stern RK, Choi K, Agresti M, Tamai I, Mehta ND, Roninson IB. Molecular basis of preferential resistance to colchicine in multidrug-resistant human cells conferred by Gly-185 Val-185 substitution in P-glycoprotein. Proc Natl Acad Sci USA 1990; 87: 7225-9.

14. Gros P, Dhir R, Croop J, Talbot F. A single amino acid substitution strongly modulates the activity and substrate specificity dulates the activity and substrate specificity
of the mouse Mdr1 and Mdr3 drug efflux pumps. Proc Natl Acad Sci USA 1991; 88: 7289-93.

15. Devine SE, Ling V, Melera PW. Amino acid substitutions in the sixth transmembrane domain of $\mathbf{P}$-glycoprotein alter multidrug resistance. Proc Natl Acad Sci USA 1992; $89: 4564-8$.

16. Loo TW, Clarke DM. Functional consequences of proline mutations in the predicted transmembrane domain of P-glycoprotein. I Biol Chem 1993; 268 : 3143-9.

17. Loo TW, Clarke DM. Functional consequences of phenylalanine mutations in the predicted transmembrane domain of P-glycoprotein. J Biol Chem 1993; 268 : 19965-72.

18. Loo TW, Clarke DM. Functional consequences of glycine mutations in the predicted cytoplasmic loops of P-glycoprotein. $J$ Biol Chem 1994; 269 : 7243-8.

19. Higgins CF. ABC transporters : from microorganisms to man. Annu Rev Cell Biol $1992 ; 8: 67-113$.

20. McGrath JP, Varshavsky A. The yeast STE6 gene encodes a homologue of the mammalian multidrug resistance P-glycoprotein. Nature 1989 ; 340 : 400-4.

21. Berkower C, Michaelis S. Mutational analysis of the yeast a-factor transporter STE6, a member of the ATP binding cassette (ABC) protein superfamily. EMBO J $1991 ; 10: 3777-85$.

22. Raymond M, Gros P, Whiteway M, Thomas DY. Functional complementation of yeast ste 6 by a mammalian multidrug resistance $m d r$ gene. Science $1992 ; 256$ : 232-4.

23. Ruetz S, Gros P. Functional expression of P-glycoprotein in secretory vesicles. $/ \mathrm{Biol}$ Chem 1994; 269 : 12277-84.

24. Riordan JR, Rommens JM, Kerem BS, Alon N, Rozmahel R, Grzelczak Z, Zielenski J, Lok S, Plavsic N, Chou JL, Drumm ML, Iannuzzi MC, Collins FS, Tsui LC. Identification of the cystic fibrosis gene: cloning and characterization of complementary DNA. Science $1989 ; 245: 1066-73$. miques et génétiques accumulées jusqu'à présent a toutefois permis de définir certains domaines fonctionnels de la P-gp. D'abord, la région hydrophile est associée à l'utilisation de l'énergie de l'ATP nécessaire à la fonction de pompe. Le marquage de la P-gp par un analogue photoactivable de l'ATP indique en effet qu'elle est capable de fixer l'ATP. Il a, de plus, été démontré que la P-gp possède une activité ATPasique [7]. Finalement, des mutations du motif Walker A des deux sites de fixation de l'ATP conduisent à une perte de fonction [8], démontrant le caractère essentiel de chacun des deux sites. Ces mutations n'empêchent toutefois pas la liaison de l'ATP, attestant qu'une étape ultérieure, probablement l'hydrolyse de l'ATP, est requise pour l'activation de la P-gp.

L'étude de chimères, produites par l'échange de régions homologues entre les différents gènes $m d r$ de souris, indique que les sites de fixation de l'ATP des trois isoformes murines, très conservés, sont interchangeables, alors que les segments TM ne peuvent être échangés sans perdre les caractéristiques fonctionnelles de l'une des deux isoformes d'origine [4]. La région hydrophobe de la P-gp semble donc impliquée dans la reconnaissance des médicaments et dans leur excrétion. Des expériences de photomarquage utilisant des analogues photoactivables des médicaments et des anticorps dirigés contre certains épitopes de la P-gp révèlent la présence de deux sites majeurs de liaison des médicaments, un dans chaque moitié de la P-gp, près des TM6 et TM12 [9, 10]. Le marquage de ces sites sur chaque moitié de la P-gp peut être inhibé de façon symétrique par la vinblastine, suggérant que des domaines de chaque moitié de la protéine s'assemblent en une structure tertiaire pour ne former qu'un seul site de liaison. Il faut cependant ajouter que ce marquage est inhibé uniquement par l'ajout de vinblastine et non par d'autres agents antitumoraux couramment utilisés lors d'expériences in vitro, comme l'adriamycine, la colchicine et l'actinomycine $\mathrm{D}$, indiquant que ce site pourrait ne refléter que l'interaction de la P-gp avec certaines classes de médicaments. La production d'une chimère dans laquelle les segments
TM3 et TM4 du gène humain MDRl ont été remplacés par ceux du gène MDR2 a mené à l'expression d'une protéine non fonctionnelle qui conserve toutefois sa capacité de fixer les analogues photoactivables des médicaments [11]. Ce résultat démontre que la liaison à la P-gp et l'excrétion sont deux étapes distinctes du flux sortant des médicaments.

L'analyse des nombreuses mutations des différentes isoformes de la P-gp a permis de révéler des acides aminés à proximité ou dans les segments TM dont la substitution modifie l'affinité et l'excrétion de certains médicaments (Tableau I). Ainsi, le remplacement d'une valine par une glycine près du segment TM3 du gène humain MDR1 (mutant V185G, Tableau I) augmente le niveau de résistance à la colchicine et à l'adriamycine alors que la résistance à la vinblastine est réduite [12].

Curieusement, la liaison d'analogues photoactivables de la vinblastine au mutant V185G est accrue par rapport à la protéine non mutée, alors que celle des analogues de la colchicine est diminuée [13], suggérant que la mutation V185G n'altère pas l'étape initiale de liaison des médicaments, mais plutôt l'étape suivante d'excrétion. Le remplacement dans le segment TMll d'une sérine par une phénylalanine des gènes $m d r l$ ou $m d r 3$ de souris (S941F et S939F, Tableau I) diminue grandement la résistance à l'adriamycine et à la colchicine, mais affecte peu la résistance à la vinblastine [14]. Cette fois, la perte de résistance du mutant $m d r 3 \mathrm{~S} 939 \mathrm{~F}$ est liée à une diminution de la liaison des analogues photoactivables de médicaments, ce qui pourrait indiquer, dans le cas du mutant S939F, une perte variable de l'affinité pour les différents médicaments, alors que la vitesse d'excrétion n'est pas affectée. Le double remplacement glycine-alanine par alanine-proline dans le segment TM6 du gène $p g p 1$ de hamster (homologue du gène murin $m d r 3$ ), surexprimé dans des cellules pulmonaires de hamster chinois sélectionnées pour la résistance à l'actinomycine D (G338A/A339P, Tableau I), conduit à une résistance accrue à l'actinomycine $\mathrm{D}$ par rapport au gène normal pgp1, mais cause, en revanche, une perte de la résistance à 
Tableau I

PHÉNOTYPE DES VARIANTS DE LA P-gP

\begin{tabular}{|lcccccc|}
\hline \multicolumn{7}{c|}{ Résistance* relative aux médicaments $^{*}$} \\
\hline Mutations & Gènes & COL & ADM & VBL & ACT & Réf \\
V185G/TM3 & MDR1 & $\uparrow 4 x$ & $\uparrow 2 x$ & $\downarrow 3 x$ & $\downarrow 4 x$ & {$[12]$} \\
S941F/TM11 & mdr1 & $\downarrow 33 x$ & $\downarrow 15 x$ & $\downarrow 4 x$ & $\downarrow 2 x$ & {$[14]$} \\
S939F/TM11 & mdr3 & $\downarrow 29 x$ & $\downarrow 14 x$ & $\downarrow 3 x$ & $\downarrow 12 x$ & {$[14]$} \\
G338A+A339P/TM6 & $p g p l$ & $\downarrow 4 x$ & $\downarrow 3 x$ & $1 x$ & $\uparrow 3 x$ & {$[15]$} \\
F335A/TM6 & & $\uparrow 1,3 x$ & $\uparrow 1,6 x$ & $\downarrow 7 x$ & $\downarrow 6 x$ & {$[17]$} \\
G187V/TM3 & & $\uparrow 3 x$ & $\uparrow 3 x$ & $1 x$ & $\downarrow 3 x$ & {$[18]$} \\
G830V/TM8 & & $\uparrow 3 x$ & $\uparrow 3 x$ & $1 x$ & $\downarrow 2 x$ & {$[18]$} \\
P223A/TM4 & MDR1 & $\downarrow 4 x$ & $\downarrow 3 x$ & $\uparrow 2 x$ & $\downarrow 4 x$ & {$[16]$} \\
F978A/TM12 & & $\downarrow 9 x$ & $\downarrow 10 x$ & $\downarrow 4 x$ & $\downarrow 4 x$ & {$[17]$} \\
F777A/TM8 & & $\downarrow 2 x$ & $\downarrow 2 x$ & $\downarrow 2 x$ & $\downarrow 2 x$ & {$[17]$} \\
\hline
\end{tabular}

* La résistance des différents mutants à la colchicine (COL), l'adriamycine (ADM), la vinblastine (VBL) et l'actinomycine $D(A C T)$ est exprimée par rapport à la résistance de la protéine normale à ces mêmes substances.

Les mutations sont notées sous une forme indiquant l'acide aminé muté (code à une lettre), le numéro du codon, le nouvel acide aminé et le numéro de l'hélice transmembranaire.

d'autres médicaments tels que la colchicine et la daunomycine [15]. Finalement, une analyse systématique de la fonction de la protéine codée par le gène $M D R l$ humain, dans laquelle on a remplacé, par mutagenèse dirigée, toutes les prolines [16], les phénylalanines [17] et les glycines [18] des régions transmembranaires, a permis de montrer que certaines mutations affectent de façon variée le profil de résistance aux différentes classes de médicaments (Tableau I). Ces résultats pourraient, soit indiquer la présence à l'intérieur de la P-gp de plusieurs sites d'interaction avec les médicaments adjacents, soit suggérer que les diverses classes de médicaments interagissent spécifiquement avec différents acides aminés à l'intérieur d'un même site d'attachement.

L'ensemble de ces analyses réalisées sur les différentes isoformes de la P-gp a donc permis de définir certaines des caractéristiques fonctionnelles de la protéine. D'abord, il est clair que la liaison et l'hydrolyse de l'ATP aux deux sites de fixation de l'ATP sont essentielles à la fonction d'excrétion des médicaments par la P-gp. Ensuite, la reconnaissance et l'excrétion des médicaments se font via les segments TM de la protéine, par la liaison des médicaments à ce qui semble être un seul ou plusieurs sites juxtaposés, formé(s) par la jonction de domaines des deux moitiés de la P-gp. De plus, la reconnaissance des médicaments MDR par les différentes isoformes de la P-gp dépend des régions TM comme l'ont démontré l'analyse des chimères $m d r 1 / m d r 2$ et l'effet de mutations ponctuelles entraînant des modifications du profil de résistance aux médicaments.

\section{Famille $A B C$}

Les sites de fixation de l'ATP de la P-gp, incluant les motifs Walker A et $B$, s'avèrent extrêmement conservés dans l'évolution et se retrouvent dans plusieurs autres protéines membranaires, tant chez les organismes procaryotes que chez les eucaryotes [19]. Ces protéines sont regroupées dans une famille de gènes désignée $A B C$ (pour ATP-binding-cassette) en raison de l'homologie de leur site de fixation de l'ATP.

Un grand nombre de transporteurs eucaryotes de la famille $\mathrm{ABC}$ possède une structure similaire à celle de la P-gp incluant, outre les deux sites de fixation de l'ATP, deux régions hydrophobes formées de segments TM. L'étude fonctionnelle de ces transporteurs $\mathrm{ABC}$ pourrait, grâce à leur homologie structurale, permettre de mieux comprendre le mécanisme d'action de la P-gp. Nous allons donc présenter succinctement les études pertinentes portant sur la relation structure/fonction des transporteurs $\mathrm{ABC}$ les plus apparentés à la P-gp: le transporteur du facteur a chez Saccharomyces cerevisiae (STE6), le canal chlorure CFTR et les cotransporteurs de peptides antigéniques, TAP1 et TAP2.

\section{STE6}

Lors du processus de conjugaison chez la levure $S$. cerevisiae, les cellules de type a sécrètent dans le milieu extracellulaire un facteur de conjugaison, le facteur a, un dodécapeptide hydrophobe prénylé et méthylé. Ce facteur est ensuite capté par un récepteur des cellules de type $\alpha$, où il déclenche une cascade de modifications cellulaires menant éventuellement à la conjugaison. Le gène STE6 code pour le transporteur membranaire du facteur a. Ce transporteur, comme la P-gp, est formé par la fusion de deux parties symétriques composées d'un site de fixation de l'ATP et d'une région de six segments TM [20]. L'invalidation du gène STE6 par recombinaison homologue chez la levure conduit à un phénotype de stérilité (souches $\Delta$ STE6). Ces souches stériles ont permis la caractérisation fonctionnelle du gène puisqu'il est possible de corriger le phénotype de stérilité par la transformation des levures avec des vecteurs permettant l'expression exogène de STE6. On a ainsi démontré que la mutation des acides aminés les plus conservés à l'intérieur des motifs Walker A et B, dans l'un ou l'autre des sites de fixation de l'ATP de STE6, empêche la complémentation de $\triangle$ STE6, démontrant la nécessité absolue des deux sites de fixation de l'ATP pour le transport du facteur a [21]. Les deux moitiés de la protéine restent aptes à transporter le facteur a, même lorsqu'elles sont exprimées séparément dans la même cellule, alors qu'une seule moitié ne suffit pas à complémenter $\triangle \mathrm{STE} 6$. Les deux demi-molécules peuvent donc s'assembler pour former un hétérodimère qui reconstitue la structure tridimensionnelle de STE6. L'expression du gène $m d r 3$ dans une telle souche stérile peut partiellement complémenter la perte du gène STE6, ce qui confirme que l'analogie de structure entre ces deux gènes, l'un de souris et l'autre de levure, se traduit par une fonction similaire. $\mathrm{Ce}$ résultat suggère un mécanisme d'ac- 


\section{RÉFÉRENCES}

25. Rich DP, Gregory RJ, Anderson MP, Manavalan P, Smith AE, Welsh MJ. Effect of deleting the $\mathbf{R}$ domain on CFTR-generated chloride channels. Science 1991 ; 253 : 205-7.

26. Férec C, Mercia B, Audrézet MP. Les mutations de la mucoviscidose : du génotype au phénotype. médecine/sciences $1994 ; 10$ : pe au phe

27. Denning GM, Ostedgaard LS, Welsh MJ. Abnormal localization of cystic fibrosis transmembrane conductance regulator in primary cultures of cystic fibrosis airway epithelia.J Cell Biol 1992; 118 : 551-9.

28. Denning GM, Anderson MP, Amara JF, Marshall J, Smith AE, Welsh MJ. Processing of mutant cystic fibrosis transmembrane conductance regulator is temperature-sensitive. Nature 1992; 358: 761-4.

29. Cheng $\mathrm{SH}$, Gregory RJ, Marshall J, PaulS, Souza DW, White GA, O'Riordan CR, Smith AE. Defective intracellular transport and processing of CFTR is the molecupor basis of most cystic fibrosis. Cell 1991; 63 : 827-34.

30. Sheppard DN, Rich DP, Ostedgaard LS, Gregory RJ, Smith AE, Welsh MJ. Mutations in CFTR associated with mild disease form $\mathrm{Cl}^{-}$channels with altered pore properties. Nature 1993 ; 362 : 160-4.

31. Parham P. Flying the first class flag. $\mathrm{Na}$ ture 1992 ; 357: 193-4.

32. Bahram S. Transporteurs de peptides et présentation de l'antigène. médecine/sciences 1993; 9 : 1204-13.

33. Spies T, Cerundolo V, Colonna $M$, Cresswell P, Townsend A, DeMars R. Presentation of viral antigen by MHC class I molecules is dependent on a putative peptide transporter heterodimer. Nature 1992 ; 355 : 644-6.

34. Kelly A, Powis SH, Kerr IA, Mockridge I, Elliott $\mathrm{T}$, Bastin J, Uchanska-Ziegler B, Ziegler A, Trowsdale J, Townsend A. Assembly and function of the two $\mathrm{ABC}$ transporter proteins encoded in the human major histocompatibility complex. Nature 1992; 355 : $641-4$

35. Powis SJ, Deverson EV, Coadwell WJ, Ciruela $A$, Huskisson NS, Smith $H$, Butcher GW, Howard JC. Effect of polymorphism of an MHC-linked transporter on the peptides assembled in a class I molecule. Nature 1992; 357: 211-5.

36. Cordon-Cardo C, O'Brien JP, Casals D, Rittman-Grauer L, Biedler JL, Melamed MR, Bertino JR. Multidrug-resistance gene (Pglycoprotein) is expressed by endothelial
cells at the bloodbrain barrier sites. Proc tion commun pour ces deux transporteurs, même si leur substrat respectif est différent. L'utilisation d'un mutant thermosensible de levure (mutant SEC6-4) défectueux au niveau de la fusion des vésicules de sécrétion à la membrane plasmique permet, à la température non permissive $\left(37^{\circ} \mathrm{C}\right)$, l'accumulation et l'obtention d'une grande quantité de vésicules de sécrétion étanches, uniformes et ayant une polarité inversée par rapport à la membrane plasmique (inside-out) $(f i-$ gure 3A). L'expression des gènes murins $m d r 1$ et $m d r 3$, et non celle de $m d r 2$, dans la membrane de vésicules de sécrétion du mutant SEC6-4 permet le transport actif de médicaments antitumoraux à l'intérieur de ces vésicules [23]. Ce transport peut être inhibé par des agents qui bloquent l'action de la P-gp dans les cellules résistantes, comme le vérapamil. Ces résultats démontrent qu'un tel système peut reproduire in vitro l'activité de la P-gp.

\section{CFTR}

La mucoviscidose ou fibrose kystique du pancréas est la maladie génétique la plus répandue dans la population d'origine européenne, affectant une personne sur 2500. Cette maladie est causée par une anomalie du transport du chlorure au niveau des cellules épithéliales et est associée à des mutations du gène $C F T R$ (cystic fibrosis transmembrane conductance regulator). La protéine CFTR possède, en plus des deux sites de fixation de l'ATP (NBF1 et NBF2 pour nucleotide binding fold) et des régions $\mathrm{TM}$, caractéristiques de la famille $\mathrm{ABC}$, un domaine de régulation (le domaine R) situé entre les deux parties symétriques de la molécule [24]. L'activité de canal chlorure de CFTR est liée à la phosphorylation de ce domaine par la protéine kinase A sous l'effet de l'AMPc [25]. La délétion du domaine $\mathrm{R}$ conduit d'ailleurs à la production d'un canal chlorure non réglé, continuellement actif et permet, de plus, la suppression de l'effet de mutations du domaine NBF2 mais non du domaine NBF1 [25]. Ces résultats suggèrent, d'une part, que le domaine $\mathrm{R}$ interagit avec le NBF2 lors de la régulation du canal chlorure et, d'autre part, que les deux sites de fixation de l'ATP de
CFTR jouent un rôle fonctionnel différent.

Plus de 400 allèles mutés du gène $C F T R$, responsables de formes plus ou moins sévères de la mucoviscidose, ont été décrits jusqu'à présent [26]. Même si les mutations sont réparties sur la presque totalité du gène, les plus communes sont situées dans les deux sites de fixation de l'ATP, et plus particulièrement dans le NBF1. Ces mutations causent les formes les plus sévères de la maladie. La délétion d'une phénylalanine en position $508(\Delta \mathrm{F} 508)$ dans le NBFl est responsable, à elle seule, de près de $70 \%$ des cas de mucoviscidose. La mutation $\Delta$ F508 entraîne une anomalie du transit cellulaire du transporteur, qui est retenu dans le réticulum endoplasmique rugueux et rapidement dégradé [27]. En revanche, l'expression de la forme mutante $\Delta$ F508 in vitro dans des cellules fibroblastiques humaines maintenues à des températures plus basses permet le ciblage d'un transporteur actif à la membrane plasmique, indiquant que $\Delta F 508$ est une mutation thermosensible n'affectant pas directement l'activité de canal chlorure de la protéine [28]. La plupart des autres mutations des NBF1 et NBF2 ne causent pas d'anomalies du transit cellulaire de la protéine. Il en résulte plutôt des canaux chlorure inactifs dans la membrane plasmique [29].

Les mutations des segments TM causent des formes moins sévères de la maladie. Les mutations d'acides aminés chargés entre les segments TM1 à TM6 altèrent la spécificité anionique du canal ou en diminuent la conductivité [30]. Le phénotype associé à ces mutations montre que les segments TM sont intimement liés au passage des ions $\mathrm{Cl}^{-}$à travers le canal. Les analyses des formes mutantes de CFTR montrent encore une fois la dichotomie des rôles entre les différentes unités structurales du transporteur $\mathrm{ABC}$ : les sites de fixation de l'ATP sont responsables de l'activation et de la régulation du transporteur, alors que les régions TM reconnaissent et/ou lient leur(s) substrat(s) spécifique(s).

\section{TAP1/TAP2}

Deux autres transporteurs ABC, TAP1 et TAP2 (pour transporter asso- 

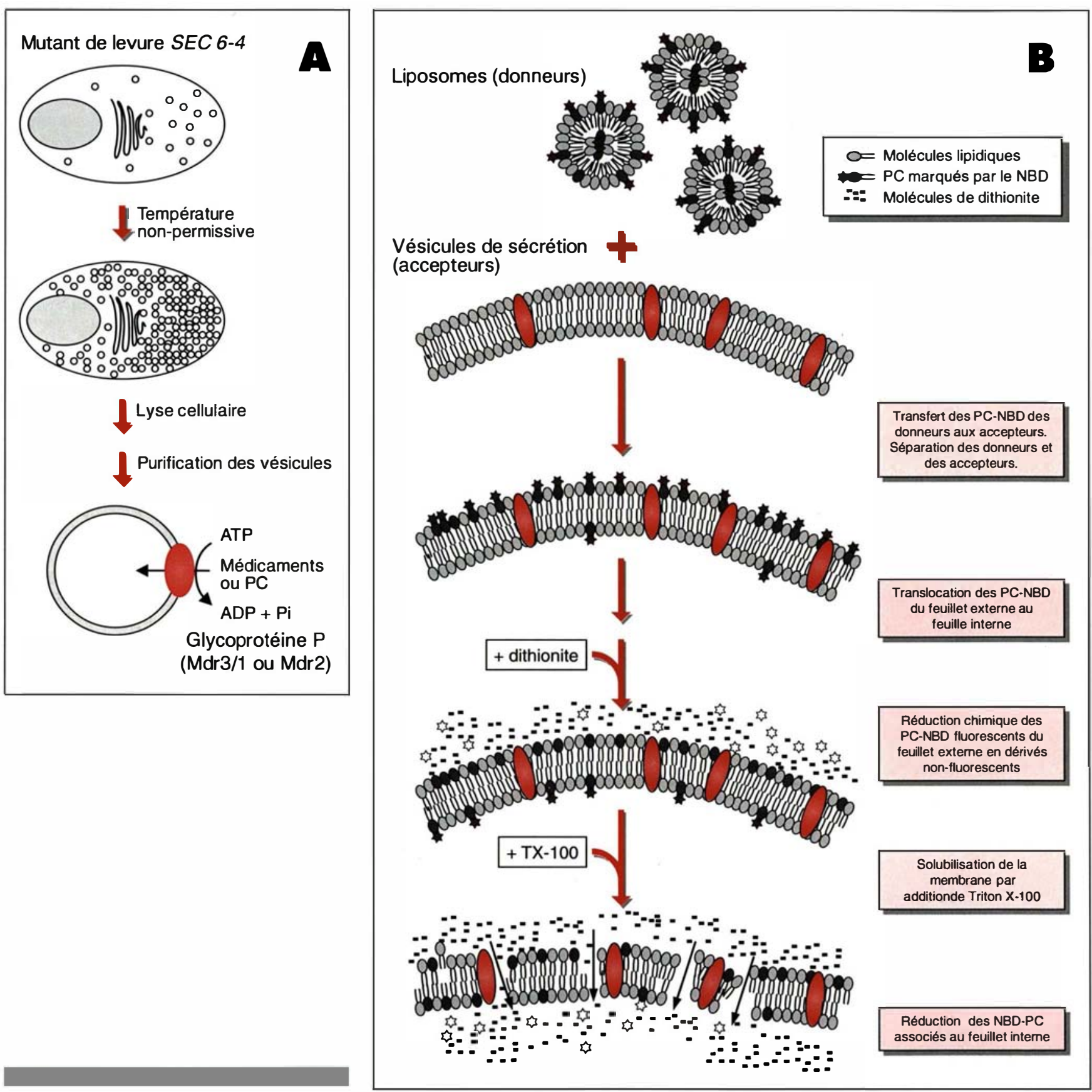

Figure 3. Expression de la glycoprotéine P dans la levure Saccharomyces cerevisiae. A. Production des vésicules de sécrétion (VS) dans le mutant de levure SEC6-4. L'isoforme de la P-gp est exprimée dans les levures qui sont alors incubées à la température permissive $\left(25^{\circ} \mathrm{C}\right)$. Lorsqu'elles sont transférées à la température non permissive $\left(37^{\circ} \mathrm{C}\right)$, ces cellules produisent une grande quantité de vésicules de sécrétion qui ne peuvent fusionner à la membrane plasmique. Ces vésicules peuvent être isolées et utilisées pour caractériser l'isoforme de la P-gp exprimée (transport de médicaments ou translocation de phosphatidylcholine). B. Méthode biochimique permettant la mesure de la translocation de la phosphatidylcholine (PC) relayée par Mdr2 dans les membranes des vésicules de sécrétion [46]. Les PC (gris foncé) marquées par le NBD (bistre) sont d'abord transférées des liposomes (donneurs) aux membranes des vésicules (accepteurs) (gris clair). Le NBD peut être réduit en un dérivé non fluorescent (blanc) en présence de dithionite. La quantité de fluorescence émise par les PC-NBD est déterminée successivement avant ajout de dithionite, après ajout de dithionite, et après solubilisation des membranes vésiculaires par le détergent Triton X-100. La différence entre le niveau de fluorescence émise avant et après solubilisation des membranes des vésicules len présence de dithionite) représente la portion des PC-NBD transférées du feuillet externe au feuillet interne. 


\section{RÉFÉRENCES}

37. Thiebault $F$, Tsuruo $T$, Hamada $H$, Gottesman MM, Pastan I, Willingham MC. Cellular localization of the multidrug-resistance gene product P-glycoprotein in normal human tissues. Proc Natl Acad Sci USA 1987. $84: 7735-8$

38. Croop JM, Raymond M, Haber D, Devault $A$, Arceci $R J$, Gros $P$, Housman DE. The three mouse multidrug resistance $(m d r)$ genes are expressed in a tissue-specific manner in normal mouse tissues. Mol Cell Biol $1989 ; 9: 1346-50$

39. Arceci RJ, Croop JM, Horwitz SB, Housman $\mathrm{DE}$. The gene encoding multidrug resistance is induced and expressed at high levels during pregnancy in the secretory epithelium of the uterus. Proc Natl Acad Sci USA 1988; 85: 4350-4.

40. Tatsuta T, Naito M, Oh-hara T, Sugawara I, Tsuoro T. Functional involvement of $\mathrm{P}$ glycoprotein in blood-brain barrier. J Biol Chem 1992; 267: 20383-91.

41. Schinkel AH, Smit JJM, van Tellingen O Beijnen JH, Wagenaar E, van Deemter L, Mol CAAM, van der Valk MA, RobanusMaandag EC, te Riele HPJ, Berns AJM Borst P. Disruption of the mouse mdrla $\mathrm{P}$ glycoprotein gene leads to a deficiency in the blood-brain barrier and to increased sensitivity to drugs. Cell $1994 ; 77: 491-502$.

42. Ueda $\mathrm{K}$, Okamura N, Hirai M, Tanigawara Y, Saeki T, Kioka N, Komano T, Hori R. Human P-glycoprotein transports cortisol, aldosterone, and dexamethasone, but not progesterone. $J$ Biol Chem 1992 $267: 24248-52$

43. Wolf DC, Horwitz SB. P-glycoprotein transports corticosterone and is photoaffinity-labeled by the steroid. Int J Cancer 1992 52: 141-6.

44. Smit JJM, Schinkel AH, Oude Elferink RPJ, Groen $A K$, Wagenaar $E$, van Deemter L, Mol CAAM, Ottenhoff R, van der Lugt NMT, van Roon MA, van der Valk MA, Offerhaus GJA, Berns AJM, Borst P. Homozygous disruption of the murine $m d r 2$ P-glycoprotein gene leads to a complete absence of phospholipid from bile and to liver disease. Cell 1993; 75: 451-62.

45. Higgins CF, Gottesman MM. Is the multidrug transporter a flippase? Trends Biochem Sci 1992; 17: 18-20.

46. Ruetz S, Gros P. Phosphatidylcholine translocase: a physiological role for the ciated with antigen processing) codés par deux gènes localisés dans la région de la classe II du complexe majeur d'histocompatibilité (CMH), ont récemment été identifiés [31, 32]. Ces protéines sont formées d'un site de fixation de l'ATP et d'une région TM. Une anomalie au niveau de l'expression d'un de ces deux gènes bloque le processus de présentation aux lymphocytes $\mathrm{T}$ cytotoxiques de peptides antigéniques associés à la classe I du CMH. Les deux transporteurs TAP1 et TAP2 s'assemblent à la membrane du réticulum endoplasmique et forment un seul complexe fonctionnel [33, 34]. Celui-ci permet la translocation de peptides antigéniques du cytosol au réticulum endoplasmique où ils sont alors associés au CMH I et acheminés à la surface des cellules. En formant un hétérodimère, TAP1 et TAP2 reconstituent l'unité fonctionnelle retrouvée dans les autres transporteurs ABC. Des variations de séquences dans les segments TM de l'isoforme TAP2 du rat sont associées à la capacité du transporteur de reconnaître des classes distinctes de peptides hydrophiles ou hydrophobes [35], démontrant une fois de plus la relation entre les segments TM et la reconnaissance du substrat.

L'ensemble de ces travaux sur la P-gp et les autres transporteurs $\mathrm{ABC}$ a mis en évidence certaines caractéristiques fonctionnelles et structurales communes à ces transporteurs. D'abord, la fonction de transporteur (ou de canal) nécessite deux unités de bases, chacune étant constituée d'un site de fixation de l'ATP et d'une région hydrophobe transmembranaire. Ensuite, non seulement les deux sites de fixation de l'ATP sont requis mais ils semblent jouer des rôles fonctionnels distincts au niveau de l'activation et de la régulation du transporteur. Finalement, les régions transmembranaires sont impliquées directement dans les interactions entre le transporteur et le substrat, soit au niveau de la reconnaissance, soit au niveau de la liaison et du transport.

\section{Fonction de la P-gp dans les tissus normaux}

L'expression des différents gènes $m d r$ dans l'organisme est sous un contrôle tissulaire précis, ce qui suggère que la $\mathrm{P}$-gp remplit des fonctions physiologiques importantes. Les hypothèses mises en avant quant à son (ses) rôle(s) dans les différents tissus tiennent compte, d'une part, de sa fonction de pompe d'excrétion dans les cellules résistantes aux médicaments, ce qui suggère un rôle de protection et, d'autre part, de sa distribution cellulaire et subcellulaire spécifique qui pourrait indiquer une implication dans le transport de certains substrats physiologiques. Les sites d'expression du gène humain $M D R 1$ chevauchent ceux des gènes murins $m d r 1$ et $m d r 3$, alors que les
Tableau II

RÔLES DES ISOFORMES MURINS DE LA P-gP DANS LES TISSUS SAINS

$\begin{array}{lll}\text { Gène Sites d'expression } & \begin{array}{l}\text { Phénotype relié } \\ \text { à l'invalidation }\end{array} & \begin{array}{l}\text { Rôle } \\ \text { (rôle possible) }\end{array}\end{array}$

Glandes surrénales

(Détoxication)

Placenta

mdr1 Rein ?

Utérus des souris gravides

mdr2 Foie

Symptomatologie Translocation des PC hépatique

Tube digestif

Poumon

mdr3 Foie

Intoxication

Barrière hémato- du cerveau

Détoxication encéphalique contre xénobiotiques 
gènes humain $M D R 2$ et murin $m d r 2$ ont une distribution tissulaire semblable. L'analyse de l'expression de l'ARN messager ou de la protéine produits par le gène humain $M D R 1$ montre un niveau maximal d'expression dans la portion médullaire des glandes surrénales, le rein, le jéjunum, le côlon, ainsi que dans les cellules endothéliales vasculaires des testicules et de la barrière hématoencéphalique, alors que l'expression de $M D R 2$ est restreinte presque exclusivement au foie $[36,37]$. Chez la souris, $m d r l$ est surtout exprimé dans les glandes surrénales, le placenta, le rein, et l'utérus des souris en gestation. L'expression de $m d r 2$ est localisée dans le foie et les muscles, et on retrouve $m d r 3$ dans le poumon, le tube digestif et la barrière hématoencéphalique [38] (Tableau II).

Les analyses immunohistochimiques démontrent que la localisation subcellulaire de la P-gp est polarisée dans les membranes apicales des cellules épithéliales recouvrant la surface luminale du jéjunum et du côlon, des tubules proximaux rénaux, des canalicules biliaires et pancréatiques, et de l'endomètre des souris en gestation [36, 37, 39]. Cette expression polarisée à la surface luminale d'organes émonctoires est compatible avec un rôle de la P-gp dans des processus de détoxication cellulaire. L'expression de la P-gp dans les cellules endothéliales de la barrière hémato-encéphalique reste cependant la plus significative pour appuyer l'hypothèse d'un rôle de détoxication de la P-gp, comme en font foi plusieurs travaux $\left(\mathrm{m} / \mathrm{s} n^{\circ} 8 / 9\right.$, vol. 10 , p. 915). D'abord, des lignées de cellules endothéliales vasculaires provenant de cerveaux de souris montrent que la P-gp est fonctionnelle dans ces cellules puisqu'elle permet le transport de la vinblastine [40]. En outre, on a démontré que ce transport est directionnel, de la surface basale à la surface apicale de ces cellules, ce qui apparaît compatible avec l'idée que la P-gp bloque l'entrée de xénobiotiques dans le cerveau. Finalement, la production de souris mutantes dont le gène $m d r 3$ (mdrla) a été invalidé par recombinaison homologue [41] révèle que l'absence de la P-gp au niveau des cellules endothéliales de la barrière hémato-encéphalique cause une augmentation dramatique de

$\mathrm{m} / \mathrm{s} n^{\circ} 3$, vol. 11 , mars 95 l'intoxication cérébrale de ces souris (mdr3-/-) par l'ivermectine, un acaricide utilisé contre la gale: le traitement des souris à l'ivermectine, normalement sans conséquence, est fatal aux souris $m d r 3-/-\left(m / s n^{\circ} 8-9\right.$, vol. 10 , p. 915). De plus, après injection par voie intraveineuse de la vinblastine à ces souris $m d r 3-/-$ on constate une altération de la distribution tissulaire et de la pharmacocinétique de cet agent antitumoral; notamment, une accumulation de vinblastine 22 fois plus élevée est détectée dans le cerveau où, de plus, son taux d'élimination est nettement réduit. Ces résultats montrent que la P-gp peut aussi agir comme une pompe d'excrétion des médicaments dans les tissus sains, en particulier au niveau de l'endothélium vasculaire de la barrière hémato-encéphalique. Par ailleurs, ils permettent d'expliquer, d'une part, l'efficacité limitée de la chimiothérapie dans le traitement des tumeurs cérébrales et, d'autre part, les effets secondaires observés chez les patients traités avec des agents antitumoraux en combinaison avec des inhibiteurs de la P-gp, tels que le vérapamil ou la ciclosporine A $\left(m / s n^{\circ} 8 / 9\right.$, vol. $10, p$. 915).

Cette démonstration remarquable d'une fonction de détoxication de la P-gp n'exclut toutefois pas que ce transporteur puisse assumer d'autres rôles dans les tissus normaux D'ailleurs, la localisation de la P-gp à des sites tels les ductules pancréatiques, l'endomètre des souris gravides et le cortex surrénalien est peu compatible avec un rôle de détoxication, mais suggère plutôt une implication dans le transport d'un substrat physiologique normal. Par exemple, il est possible que l'isoforme de la P-gp produite par le gène $m d r l$ puisse être un transporteur d'hormones stéroïdes. Des travaux ont démontré que cette isoforme peut transporter certaines hormones stéroïdes telles que le cortisol, l'aldostérone, l'hormone synthétique dexaméthasone [42], de même que la corticostérone [43]. Par ailleurs, la capacité des cellules exprimant la P-gp de complémenter la déficience de transport du facteur a de la levure [22], un dodécapeptide, suggère un rôle possible dans la translocation d'hormones peptidiques.

La démonstration la plus probante d'une fonction physiologique normale associée à une isoforme de la P-gp a été obtenue par l'étude du gène murin $m d r 2$ exprimé au niveau des canalicules biliaires. La production de souris mutantes dont le gène $m d r 2$ a été invalidé ( $m d r 2-/-)$ montre qu'en l'absence de cette isoforme une affection hépatique sévère se développe $\left(m / s n^{\circ} 8-9\right.$, vol. $\left.10, p .915\right)$ [44] Cette affection, caractérisée par un syndrome cholestatique avec hyperbilirubinémie, inclut des anomalies au niveau des hépatocytes et des ductules biliaires. L'absence complète de phospholipides (PL) dans la bile des souris homozygotes $m d r 2-/-$ semble être la cause primaire des anomalies observées. La concentration en sels biliaires dans la bile de souris est normalement très élevée et, si les micelles formées par ces sels ne peuvent incorporer des PL (en particulier, les phosphatidylcholines ou PC) dans la bile, elles auront tendance à les recruter des membranes environnantes, causant une destruction progressive des cellules des ductules et canalicules biliaires comme on peut l'observer chez les souris $m d r 2-/-$. On a donc proposé que l'isoforme Mdr2 faciliterait la translocation des PC du feuillet interne au feuillet externe de la membrane cellulaire des canalicules, permettant un apport constant en PC au niveau du feuillet externe et leur recrutement par les sels biliaires sécrétés par ces mêmes canalicules. Cela suggère, une fois de plus, un rôle de protection pour une isoforme de la P-gp, mais cette fois-ci au cours d'un processus physiologique normal. De plus, cette fonction de Mdr2 indique une activité translocase ou "flippase", qui avait préalablement été suggérée comme mécanisme d'action possible de la P-gp [45]. Cette activité "flippase" de Mdr2 a par ailleurs été détectée in vitro dans un système faisant appel à l'expression hétérologue de cette isoforme de la P-gp dans la souche mutante de levure SEC6-4 (décrite plus haut et $f i$ gure 3A) [46]. L'activité de Mdr2 a été analysée dans des vésicules de sécrétion du mutant SEC6-4 auxquelles on a ajouté des PC fluorescentes (PC-NBD) (figure 3B). L'expression du gène $m d r 2$ dans ces vésicules de sécrétion/PC-NBD, et non celle de $m d r 3$, permet la translocation des PC-NBD du feuillet externe au 
feuillet interne des vésicules. Ce résultat démontre que l'isoforme Mdr2 peut effectivement faciliter le transfert des PC d'un feuillet à l'autre d'une membrane cellulaire. Non seulement ce travail conforte l'hypothèse d'une activité de type "flippase" de Mdr2 au niveau des hépatocytes, mais il ouvre un nouveau champ d'étude pour les autres isoformes de la P-gp, voire même pour d'autres transporteurs $\mathrm{ABC}$, qui pourraient avoir un mécanisme d'action similaire à l'isoforme Mdr2. En effet, il est possible que ces transporteurs soient aussi des translocases facilitant le passage de substrats spécifiques d'un feuillet à l'autre de cette barrière physico-chimique que représente la membrane plasmique

\section{TIRÉS À PART}

P. Gros.

\section{Summary}

P glycoprotein : from multidrug resistance to lipid transport

Emergence of anticancer drug resistance in tumor cells is caused by the expression of a membrane glycoprotein named P-glycoprotein (P-gp). Although the precise mechanism of action of P-gp remains debated, its role in drug resistance has been clearly established in transfection experiments. The predicted structure of P-gp which includes two sets of six transmembrane domains and one nucleotide binding site suggests that it may function as an ATP-dependent drug transporter. The structural units of P-gp are conserved in a large number of membrane transporters, both in prokaryotes and eukaryotes, which together form the ATP-binding cassette (ABC) superfa- mily. The structural similarities between P-gp and its closest homologs of the $\mathrm{ABC}$ family sometimes translate into functional similarities. The molecular and biochemical analysis of these homologs may therefore shed light on the mechanism of action of P-gp in drug transport. This review summarizes recent findings concerning structural and functional aspects of P-gp, with respect to its role as a drug transporter in resistant cells and its possible roles in normal tissues. We also present recent work on P-gp related $\mathrm{ABC}$ members and attempt to draw general conclusions on common aspects of substrate recognition and transport by these proteins. 